PROCEEDINGS OF THE

AMERICAN MATHEMATICAL SOCIETY

Volume 134, Number 2, Pages 333-341

S 0002-9939(05)08029-9

Article electronically published on June 14, 2005

\title{
A FORMULA AND A CONGRUENCE FOR RAMANUJAN'S $\tau$-FUNCTION
}

\author{
MATTHEW PAPANIKOLAS
}

(Communicated by Wen-Ching Winnie Li)

\begin{abstract}
We determine formulas for Ramanujan's $\tau$-function and for the coefficients of modular forms on $\Gamma_{0}(2)$ in terms of finite field ${ }_{3} F_{2}$-hypergeometric functions. Using these formulas we obtain a new congruence of $\tau(p)(\bmod 11)$.
\end{abstract}

\section{INTRODUCTION}

Ramanujan's $\tau$-function is defined as the coefficients of the power series in $q$,

$$
\Delta:=q \prod_{m=1}^{\infty}\left(1-q^{m}\right)^{24}=\sum_{n=1}^{\infty} \tau(n) q^{n} .
$$

After setting $q=e^{2 \pi i z}$, the function $\Delta(z)$ is the unique normalized cusp form of weight 12 for the full modular group $\mathrm{SL}_{2}(\mathbb{Z})$.

The function $\tau(n)$ possesses many fascinating arithmetic properties, and Ramanujan [8, 10] showed that $\tau(n)$ satisfies several congruences modulo powers of $2,3,5,7,23$, and 691 . Notably

$$
\tau(n) \equiv \sigma_{11}(n) \quad(\bmod 691), \quad \forall n \geq 1,
$$

where $\sigma_{11}(n)$ is the sum of the eleventh powers of the divisors of $n$.

Based on numerical evidence, D. H. Lehmer 6 conjectured that $\tau(n) \neq 0$ for any $n$. Lehmer's conjecture remains open. Somewhat weaker, one may consider whether $\tau(p) \equiv 0(\bmod p)$ for any prime $p$, and one indeed finds that

$$
\tau(p) \equiv 0 \quad(\bmod p), \quad p=2,3,5,7,2411,
$$

but that these are the only primes $p \leq 10^{7}$ for which this congruence holds [4].

In this paper, we consider traces of Hecke operators on spaces of cusp forms of level 2, starting with a formula obtained in 3 . We obtain new formulas and congruences for certain cusp forms in terms of Gaussian ${ }_{3} F_{2}$-hypergeometric functions, and in particular prove a new formula and a new congruence for $\tau(p)$.

Received by the editors April 27, 2004 and, in revised form, September 9, 2004.

2000 Mathematics Subject Classification. Primary 11F30; Secondary 11F33, 11T24, 33C99.

This research was supported by NSF grant DMS-0340812 and NSA grant MDA904-03-1-0019. 
Theorem 1.1. Let $p$ be an odd prime. Choose $a, b \geq 0$ satisfying $p=a^{2}+b^{2}$, if $p \equiv 1(\bmod 4)$, or $a=b=0$, if $p \equiv 3(\bmod 4)$. Then

$$
\begin{aligned}
\tau(p)=-1-\left(1+\frac{3}{2} \phi(-1)\right) p^{5}+40 p^{3} a^{2} b^{2}-128 p a^{4} b^{4} & \\
& -\frac{1}{2} \sum_{\lambda=2}^{p-1} R_{12}\left(p, \phi(1-\lambda) p^{2}{ }_{3} F_{2}(\lambda)\right),
\end{aligned}
$$

where $R_{12}(p, x)=x^{5}-4 p x^{4}+2 p^{2} x^{3}+5 p^{3} x^{2}-2 p^{4} x-p^{5}$.

Here $\phi=(\dot{\bar{p}})$ is the Legendre symbol modulo $p$, and ${ }_{3} F_{2}(\lambda)$ is the value of a finite field hypergeometric function defined in $\$ 2$.

Remark 1.2. One finds that $p^{2}{ }_{3} F_{2}(\lambda) \in \mathbb{Z}$ for all $\lambda \in \mathbb{F}_{p}$ (see $\oint_{2}$, in particular (66)). Reducing the above formula modulo $p$ reveals immediately the congruence

$$
\tau(p) \equiv-1-\frac{1}{2} p^{10} \sum_{\lambda=2}^{p-1} \phi(1-\lambda){ }_{3} F_{2}(\lambda)^{5} \quad(\bmod p), \quad \forall p \geq 3 \text { prime. }
$$

Computing values of ${ }_{3} F_{2}(\lambda)$ is simple in principle but is computationally intensive for large primes. Thus unfortunately this congruence does not directly lead to improvements on the bounds in (2).

The formula from Theorem 1.1 also simplifies nicely modulo 11 . As usual, $(\dot{\overline{11}})$ denotes the Legendre symbol modulo 11.

Corollary 1.3. Let $p$ be an odd prime. Choose $a, b \geq 0$ satisfying $p=a^{2}+b^{2}$, if $p \equiv 1(\bmod 4)$, or $a=b=0$, if $p \equiv 3(\bmod 4)$. Then

$$
\begin{aligned}
\tau(p) \equiv-1-\left(1+\frac{3}{2} \phi(-1)\right) & \left(\frac{p}{11}\right)+7 p^{3} a^{2} b^{2}-7 p a^{4} b^{4} \\
& -\frac{1}{2} \sum_{\lambda=2}^{p-1}\left(\frac{\phi(1-\lambda) p^{2}{ }_{3} F_{2}(\lambda)-3 p}{11}\right) \quad(\bmod 11) .
\end{aligned}
$$

Remark 1.4. The formula for $\tau(p)$ in Theorem 1.1 can also be interpreted in terms of counting points over $\mathbb{F}_{p}$ on certain varieties defined over $\mathbb{Q}$ (cf. 3, §3]). In forthcoming work, K. Paranjape and D. Ramakrishnan have shown that $\tau(p)$ can be used to count points over $\mathbb{F}_{p}$ on a particular 11-dimensional Calabi-Yau variety. It would be interesting to compare these two formulations.

After defining Gaussian hypergeometric functions and noting some of their basic properties in 92 , we recall in $\$ 3$ a formula for the Hecke trace $\operatorname{Tr}_{k}\left(\Gamma_{0}(2), p\right)$ from [3] and derive a reformulation of it in terms of hypergeometric functions (see Theorem 3.2). Theorem 1.1 is an application of Theorem 3.2. In $\$ 4$ we use Theorem 3.2 to prove a general congruence for traces of Hecke operators on $\Gamma_{0}(2)$ (see Theorem 4.1), and we obtain Corollary 1.3 as an immediate consequence. We also use these methods to find formulas and congruences for coefficients of other modular forms (see Theorem 3.4 and Corollary 4.2).

\section{Gaussian hypergeometric functions}

Following the definitions of J. Greene 5 we define hypergeometric functions over finite fields that are sums of products of Jacobi sums. Let $\mathbb{F}_{q}$ denote the finite field with $q$ elements, where $q$ is a power of a fixed prime $p$. If $A$ is any multiplicative 
character on $\mathbb{F}_{q}^{\times}$, we extend it to $A: \mathbb{F}_{q} \rightarrow \mathbb{C}$ by setting $A(0)=0$. Then for characters $A, B$ on $\mathbb{F}_{q}^{\times}$we define

$$
\left(\begin{array}{l}
A \\
B
\end{array}\right):=\frac{B(-1)}{q} J(A, \bar{B})=\frac{1}{q} \sum_{t \in \mathbb{F}_{q}} A(t) \bar{B}(t-1) .
$$

Given characters $A_{0}, \ldots, A_{n}$ and $B_{1}, \ldots, B_{n}$ on $\mathbb{F}_{q}^{\times}$, we define for $\lambda \in \mathbb{F}_{p}$

$$
{ }_{n+1} F_{n}\left(\begin{array}{cccc}
A_{0} & A_{1} & \cdots & A_{n} \\
& B_{1} & \cdots & B_{n}
\end{array} \mid \lambda\right):=\frac{p}{p-1} \sum_{\chi}\left(\begin{array}{c}
A_{0} \chi \\
\chi
\end{array}\right)\left(\begin{array}{c}
A_{1} \chi \\
B_{1} \chi
\end{array}\right) \cdots\left(\begin{array}{c}
A_{n} \chi \\
B_{n} \chi
\end{array}\right) \chi(\lambda),
$$

where $\chi$ runs over all characters on $\mathbb{F}_{p}^{\times}$. Using the classical hypergeometric functions as a guide, Greene showed that these hypergeometric functions satisfy many interesting transformation laws and presented evidence that these functions serve as useful analogues for hypergeometric functions over finite fields.

Throughout this paper we will consider the following specialization of these hypergeometric functions. Let $p$ be an odd prime. Let $\varepsilon$ be the trivial character on $\mathbb{F}_{p}^{\times}$, and let $\phi=(\dot{\bar{p}})$ denote the unique quadratic character on $\mathbb{F}_{p}^{\times}$. For $\lambda \in \mathbb{F}_{p}$ we set

$$
{ }_{n+1} F_{n}(\lambda):={ }_{n+1} F_{n}\left(\begin{array}{cccc|c}
\phi & \phi & \cdots & \phi \\
& \varepsilon & \cdots & \varepsilon & \lambda
\end{array}\right)=\frac{p}{p-1} \sum_{\chi}\left(\begin{array}{c}
\phi \chi \\
\chi
\end{array}\right)^{n+1} \chi(\lambda) .
$$

Using basic properties of Jacobi sums (cf. [5, §3]), it follows that for all $\lambda \in \mathbb{F}_{p}$,

$$
p^{n} \cdot{ }_{n+1} F_{n}(\lambda) \in \mathbb{Z}
$$

Values of $p^{n} \cdot{ }_{n+1} F_{n}(\lambda)$ are closely connected to various arithmetic objects such as the traces of Frobenius of elliptic curves [7, counting points on varieties over finite fields [1, 2, [3] and the coefficients of modular forms [1], 2], 3].

In particular, if we consider the family of elliptic curves

$$
E(\lambda): y^{2}=(x-1)\left(x^{2}+\lambda\right),
$$

then after setting

$$
A(\lambda):=p+1-|E(\lambda)|,
$$

we have the following result of $\mathrm{K}$. Ono 7 .

Theorem 2.1 (Ono [7, Thm. 5]). For an odd prime $p$ and $\lambda \in \mathbb{F}_{p} \backslash\{0,-1\}$,

$$
p_{3}^{2}{ }_{3} F_{2}\left(1+\frac{1}{\lambda}\right)=\phi(-\lambda)\left(A(\lambda)^{2}-p\right) \text {. }
$$

\section{Traces of Hecke operators on $S_{k}\left(\Gamma_{0}(2)\right)$}

For positive integers $k$ and $N$, we let $S_{k}\left(\Gamma_{0}(N)\right)$ denote the complex vector space of cusp forms of weight $k$ and level $N$ with trivial character. For any integer $n$, we let $\operatorname{Tr}_{k}\left(\Gamma_{0}(N), n\right)$ denote the trace of the $n$-th Hecke operator $T_{n}$ acting on $S_{k}\left(\Gamma_{0}(N)\right)$.

Let $k \geq 4$ be even, and let $p$ be an odd prime. Throughout we will use the following convention: we choose integers $a, b \geq 0$ by $p=a^{2}+b^{2}$ if $p \equiv 1(\bmod 4)$, 
and by $a=b=0$ if $p \equiv 3(\bmod 4)$. We set

$$
G_{k}(p, s):=\sum_{j=0}^{\frac{k}{2}-1}(-1)^{j}\left(\begin{array}{c}
k-2-j \\
j
\end{array}\right) p^{j} s^{k-2-2 j}
$$

and

$$
\delta_{k}(p):=\left\{\begin{array}{lll}
\frac{1}{2} G_{k}(p, 2 a)+\frac{1}{2} G_{k}(p, 2 b) & \text { if } p \equiv 1 \quad(\bmod 4) \\
(-p)^{\frac{k}{2}-1} & \text { if } p \equiv 3 \quad(\bmod 4) .
\end{array}\right.
$$

The following theorem is the starting point for our considerations.

Theorem 3.1 (Frechette-Ono-Papanikolas [3, Thm. 2.3]). For a prime $p \geq 3$ and $k \geq 4$ even,

$$
\operatorname{Tr}_{k}\left(\Gamma_{0}(2), p\right)=-2-\delta_{k}(p)-\sum_{\lambda=1}^{p-2} G_{k}(p, A(\lambda)) .
$$

For integers $d, r \geq 0$, we define $c_{d}(r)$ by the generating function

$$
\frac{x+1}{\left(x^{2}+x+1\right)^{d+1}}=\sum_{j=-d}^{\infty} c_{d}(d+j) x^{j} .
$$

Then, for $k \geq 2$ even, define polynomials

$$
R_{k}(p, x):=\sum_{d=0}^{\frac{k}{2}-1} c_{d}\left(\frac{k}{2}-1\right) p^{\frac{k}{2}-1-d} x^{d} .
$$

We now state our main theorem, which expresses $\operatorname{Tr}_{k}\left(\Gamma_{0}(2), p\right)$ in terms of ${ }_{3} F_{2^{-}}$ hypergeometric functions.

Theorem 3.2. For $p \geq 3$ prime and $k \geq 4$ even,

$$
\operatorname{Tr}_{k}\left(\Gamma_{0}(2), p\right)=-2-\delta_{k}(p)-\sum_{\lambda=2}^{p-1} R_{k}\left(p, \phi(1-\lambda) p^{2}{ }_{3} F_{2}(\lambda)\right) .
$$

Before proving this theorem we require a combinatorial lemma.

Lemma 3.3. For all non-negative integers $d, r$, with $r \geq d$,

$$
c_{d}(r)=\sum_{j=0}^{r-d}(-1)^{j}\left(\begin{array}{c}
2 r-j \\
j
\end{array}\right)\left(\begin{array}{c}
r-j \\
d
\end{array}\right) .
$$

Proof. We consider the generating function defined by the right-hand side of the proposed formula,

$$
H_{d}(x):=\sum_{r=d}^{\infty} \sum_{j=0}^{r-d}(-1)^{j}\left(\begin{array}{c}
2 r-j \\
j
\end{array}\right)\left(\begin{array}{c}
r-j \\
d
\end{array}\right) x^{r-d} .
$$


Taking $m=r-d-j$, we have

$$
\begin{aligned}
H_{d}(x) & =\sum_{m=0}^{\infty} \sum_{j=0}^{\infty}(-1)^{j}\left(\begin{array}{c}
2 d+2 m+j \\
j
\end{array}\right)\left(\begin{array}{c}
d+m \\
d
\end{array}\right) x^{m+j} \\
& =\frac{1}{(x+1)^{2 d+1}} \sum_{m=0}^{\infty}\left(\begin{array}{c}
d+m \\
m
\end{array}\right) \frac{x^{m}}{(x+1)^{2 m}} \\
& =\frac{1}{(x+1)^{2 d+1}}\left(1-\frac{x}{(x+1)^{2}}\right)^{-(d+1)} \\
& =\frac{x+1}{\left(x^{2}+x+1\right)^{d+1}}
\end{aligned}
$$

where in the second and third equalities we have used the binomial expansion $(x+1)^{-(e+1)}=\sum_{j=0}^{\infty}(-1)^{j}\left(\begin{array}{c}e+j \\ j\end{array}\right) x^{j}$.

Proof of Theorem 3.2. Let $t_{k}(p):=\operatorname{Tr}_{k}\left(\Gamma_{0}(2), p\right)+2+\delta_{k}(p)$. By substituting (9) into Theorem 3.1 and then applying Theorem 2.1 we have

$$
t_{k}(p)=-\sum_{\lambda=1}^{p-2} \sum_{j=0}^{\frac{k}{2}-1}(-1)^{j}\left(\begin{array}{c}
k-2-j \\
j
\end{array}\right) p^{j}\left(p+\phi(-\lambda) p^{2}{ }_{3} F_{2}\left(1+\frac{1}{\lambda}\right)\right)^{\frac{k}{2}-1-j} .
$$

We replace $1+\frac{1}{\lambda}$ with $\lambda$ and expand to obtain

$$
\begin{aligned}
t_{k}(p)=-\sum_{j=0}^{\frac{k}{2}-1} \sum_{d=0}^{\frac{k}{2}-1-j} \sum_{\lambda=2}^{p-1}(-1)^{j}\left(\begin{array}{c}
k-2-j \\
j
\end{array}\right) & \left(\begin{array}{c}
\frac{k}{2}-1-j \\
d
\end{array}\right) \\
& \times p^{\frac{k}{2}-1-d}\left(\phi(1-\lambda) p^{2}{ }_{3} F_{2}(\lambda)\right)^{d} \\
=-\sum_{d=0}^{\frac{k}{2}-1}\left[\sum_{j=0}^{\frac{k}{2}-1-d}(-1)^{j}\left(\begin{array}{c}
k-2-j \\
j
\end{array}\right)\right. & \left.\left(\begin{array}{c}
\frac{k}{2}-1-j \\
d
\end{array}\right)\right] \\
& \times p^{\frac{k}{2}-1-d} \sum_{\lambda=2}^{p-1}\left(\phi(1-\lambda) p^{2}{ }_{3} F_{2}(\lambda)\right)^{d} .
\end{aligned}
$$

By Lemma 3.3 we are done.

We now consider applications of Theorem 3.2 to modular forms of level 2 of small weight. Specifically, from [9, Prop. 1.43 and Thm. 2.24], we see that $\operatorname{dim} S_{k}\left(\Gamma_{0}(2)\right)=$ 0 if $k=2,4,6$; also,

$$
\begin{gathered}
\operatorname{dim} S_{8}\left(\Gamma_{0}(2)\right)=1, \\
\operatorname{dim} S_{10}\left(\Gamma_{0}(2)\right)=1, \\
\operatorname{dim} S_{12}\left(\Gamma_{0}(2)\right)=2 .
\end{gathered}
$$


Of note is that $\Delta(z)$ and $\Delta(2 z)$ form a basis of $S_{12}\left(\Gamma_{0}(2)\right)$, and in particular $S_{12}\left(\Gamma_{0}(2)\right)$ contains no newforms. We let

$$
\begin{aligned}
& \sum_{n=1}^{\infty} \alpha(n) q^{n}=q \prod_{m=1}^{\infty}\left(1-q^{m}\right)^{8}\left(1-q^{2 m}\right)^{8} \in S_{8}\left(\Gamma_{0}(2)\right), \\
& \sum_{n=1}^{\infty} \beta(n) q^{n} \in S_{10}\left(\Gamma_{0}(2)\right)
\end{aligned}
$$

be the unique newforms on $\Gamma_{0}(2)$ of weights 8 and 10, respectively. As companions to Theorem 1.1. we have formulas for the coefficients of these forms.

Theorem 3.4. Let $p$ be an odd prime. Choose $a, b \geq 0$ satisfying $p=a^{2}+b^{2}$, if $p \equiv 1(\bmod 4)$, or $a=b=0$, if $p \equiv 3(\bmod 4)$.

(a) We have

$$
\alpha(p)=-2-(1+2 \phi(-1)) p^{3}+16 p a^{2} b^{2}-\sum_{\lambda=2}^{p-1} R_{8}\left(p, \phi(1-\lambda) p^{2}{ }_{3} F_{2}(\lambda)\right),
$$

where $R_{8}(p, x)=x^{3}-2 p x^{2}-p^{2} x+p^{3}$.

(b) We have

$$
\begin{aligned}
\beta(p)=-2-(3+2 \phi(-1)) p^{4}+80 p^{2} a^{2} b^{2}-256 a^{4} b^{4} & \\
& -\sum_{\lambda=2}^{p-1} R_{10}\left(p, \phi(1-\lambda) p^{2}{ }_{3} F_{2}(\lambda)\right),
\end{aligned}
$$

where $R_{10}(p, x)=x^{4}-3 p x^{3}+3 p^{3} x$.

Proofs of Theorems 1.1 and 3.4. We apply Theorem 3.2 using $\operatorname{Tr}_{8}\left(\Gamma_{0}(2), p\right)=\alpha(p)$, $\operatorname{Tr}_{10}\left(\Gamma_{0}(2), p\right)=\beta(p)$, and $\operatorname{Tr}_{12}\left(\Gamma_{0}(2), p\right)=\frac{1}{2} \tau(p)$. We first observe that when $p \equiv 1(\bmod 4)$, it follows from (9) and (10) that

$$
\begin{aligned}
\delta_{8}(p) & =3 p^{3}-16 p a^{2} b^{2}, \\
\delta_{10}(p) & =5 p^{4}-80 p^{2} a^{2} b^{2}+256 a^{4} b^{4}, \\
\delta_{12}(p) & =5 p^{5}-80 p^{3} a^{2} b^{2}+256 p a^{4} b^{4} .
\end{aligned}
$$

Thus to complete the proof, we compute the array of numbers $c_{d}(r)$ for $0 \leq d \leq$ $r \leq 5$ :

\begin{tabular}{|rrrrrr|}
\hline$c_{0}(r)$ & $c_{1}(r)$ & $c_{2}(r)$ & $c_{3}(r)$ & $c_{4}(r)$ & $c_{5}(r)$ \\
\hline 1 & & & & & \\
0 & 1 & & & & \\
-1 & -1 & 1 & & & \\
1 & -1 & -2 & 1 & & \\
0 & 3 & 0 & -3 & 1 & \\
-1 & -2 & 5 & 2 & -4 & 1 \\
\hline
\end{tabular}

4. Congruences for $\operatorname{Tr}_{k}\left(\Gamma_{0}(2), p\right)$

In this section we investigate further congruence properties of $\operatorname{Tr}_{k}\left(\Gamma_{0}(2), p\right)$ and prove the following theorem. 
Theorem 4.1. Let $\ell \geq 3$ be prime, let $n$ be a positive integer, and let $k=\ell^{n}+1$. Finally let $p \geq 3$ be prime.

(a) If $n$ is odd,

$$
\operatorname{Tr}_{k}\left(\Gamma_{0}(2), p\right) \equiv-2-\delta_{k}(p)-\sum_{\lambda=2}^{p-1}\left(\frac{\phi(1-\lambda) p^{2}{ }_{3} F_{2}(\lambda)-3 p}{\ell}\right) \quad(\bmod \ell),
$$

where $(\dot{\bar{\ell}})$ denotes the Legendre symbol modulo $\ell$.

(b) If $n$ is even,

$$
\operatorname{Tr}_{k}\left(\Gamma_{0}(2), p\right) \equiv-2-\delta_{k}(p)-\sum_{\lambda=2}^{p-1} \varepsilon_{\ell}\left(\phi(1-\lambda) p^{2}{ }_{3} F_{2}(\lambda)-3 p\right) \quad(\bmod \ell),
$$

where $\varepsilon_{\ell}$ is the trivial character modulo $\ell$.

Corollary 1.3 is an immediate application of this theorem. We also obtain corollaries for $\alpha(p)$ modulo 7 and $\beta(p)$ modulo 3 .

Corollary 4.2. Let $p$ be an odd prime. Choose $a, b \geq 0$ satisfying $p=a^{2}+b^{2}$, if $p \equiv 1(\bmod 4)$, or $a=b=0$, if $p \equiv 3(\bmod 4)$.

(a) For $p \geq 3$, we have

$$
\begin{aligned}
\alpha(p) \equiv-2-(1+2 \phi(-1)) & \left(\frac{p}{7}\right)+2 p a^{2} b^{2} \\
& -\sum_{\lambda=2}^{p-1}\left(\frac{\phi(1-\lambda) p^{2}{ }_{3} F_{2}(\lambda)-3 p}{7}\right) \quad(\bmod 7) .
\end{aligned}
$$

(b) For $p \geq 5$, we have

$\beta(p) \equiv-(1+\phi(-1))(1+\phi(3))-\left|\left\{\lambda \in \mathbb{F}_{p} \backslash\{0,1\} \mid{ }_{3} F_{2}(\lambda) \not \equiv 0(\bmod 3)\right\}\right| \quad(\bmod 3)$.

Proofs of Corollaries 1.3 and 4.2. The congruences for $\alpha(p)$ and $\tau(p)$ follow from part (a) of Theorem 4.1, using Euler's criterion to calculate $\delta_{k}(p)(\bmod \ell)$ via (16). The congruence for $\beta(p)$ follows from part (b) of Theorem 4.1 and the formula

$$
2+\delta_{10}(p) \equiv\left\{\begin{array}{lll}
1 & (\bmod 3), & \text { if } p \equiv 1 \quad(\bmod 12), \\
0 & (\bmod 3), & \text { otherwise }(p \neq 3),
\end{array}\right.
$$

which is straightforward to check.

The proof of Theorem 4.1 relies on special properties of the polynomials

$$
R_{k}(x):=R_{k}(1, x)=\sum_{d=0}^{\frac{k}{2}-1} c_{d}\left(\frac{k}{2}-1\right) x^{d} .
$$

We proceed by proving a series of lemmas.

Lemma 4.3. Define $H(x, t)$ by

$$
H(x, t):=\sum_{k \geq 2, \text { even }} R_{k}(x) t^{\frac{k}{2}-1} .
$$

Then

$$
H(x, t)=\frac{t+1}{t^{2}+(1-x) t+1} .
$$


Proof. By applying Lemma 3.3 and setting $k=2 n+2$, we have

$$
\begin{aligned}
H(x, t) & =\sum_{n=0}^{\infty} \sum_{d=0}^{n} \sum_{j=0}^{n-d}(-1)^{j}\left(\begin{array}{c}
2 n-j \\
j
\end{array}\right)\left(\begin{array}{c}
n-j \\
d
\end{array}\right) x^{d} t^{n} \\
& =\sum_{n=0}^{\infty} \sum_{j=0}^{n}(-1)^{j}\left(\begin{array}{c}
2 n-j \\
j
\end{array}\right) t^{n} \sum_{d=0}^{n-j}\left(\begin{array}{c}
n-j \\
d
\end{array}\right) x^{d} \\
& =\sum_{n=0}^{\infty} \sum_{j=0}^{n}(-1)^{j}\left(\begin{array}{c}
2 n-j \\
j
\end{array}\right) t^{n}(x+1)^{n-j} .
\end{aligned}
$$

We reorder the sum and, after setting $n=m+j$, we find that

$$
\begin{aligned}
H(x, t) & =\sum_{j=0}^{\infty}(-1)^{j} t^{j} \sum_{m=0}^{\infty}\left(\begin{array}{c}
2 m+j \\
j
\end{array}\right)(x+1)^{m} t^{m} \\
& =\sum_{m=0}^{\infty}(x+1)^{m} t^{m} \sum_{j=0}^{\infty}(-1)^{j}\left(\begin{array}{c}
2 m+j \\
j
\end{array}\right) t^{j} \\
& =\sum_{m=0}^{\infty}(x+1)^{m} t^{m}(t+1)^{-2 m-1} \\
& =\frac{t+1}{t^{2}+(1-x) t+1} .
\end{aligned}
$$

Lemma 4.4. Let $k \geq 2$ be even. Then

$$
R_{k}(x)=\prod_{j=1}^{\frac{k}{2}-1}\left(x-\left(\zeta^{j}+\zeta^{-j}+1\right)\right),
$$

where $\zeta:=\zeta_{k-1}=\exp \left(\frac{2 \pi i}{k-1}\right)$.

Proof. By Lemma 4.3,

$$
\begin{aligned}
\sum_{k \geq 2 \text { even }} R_{k}\left(x+x^{-1}+1\right) t^{\frac{k}{2}-1} & =H\left(x+x^{-1}+1, t\right) \\
& =\frac{t+1}{(t-x)\left(t-\frac{1}{x}\right)} \\
& =\sum_{k \geq 2 \text { even }} \frac{1}{x^{\frac{k}{2}-1}} \cdot \frac{x^{k-1}-1}{x-1} \cdot t^{\frac{k}{2}-1} .
\end{aligned}
$$

Therefore, for any $j$ with $1 \leq j \leq \frac{k}{2}-1, \zeta^{j}+\zeta^{-j}+1$ is a root of $R_{k}(x)$. Since $R_{k}(x)$ is monic and has degree $\frac{k}{2}-1$, the lemma holds.

Remark 4.5. It follows from Lemma 4.4 that the splitting field of $R_{k}(x)$ is the maximal real subfield of the cyclotomic field $\mathbb{Q}\left(\zeta_{k-1}\right)$.

Lemma 4.6. Let $\ell \geq 3$ be prime, let $n$ be a positive integer, and let $k=\ell^{n}+1$. Then

$$
R_{k}(x) \equiv(x-3)^{\frac{\ell^{n}-1}{2}} \quad(\bmod \ell) .
$$


Proof. The product in Lemma 4.4 holds in $\mathbb{Q}\left(\zeta_{\ell^{n}}\right)$, in which $\ell$ is totally ramified. The lemma then follows by reducing the product for $R_{k}(x)$ modulo the prime above $\ell$ in $\mathbb{Q}\left(\zeta_{\ell^{n}}\right)$.

Proof of Theorem 4.1. Since $R_{k}(p, x)$ is the homogenization of $R_{k}(x)$, Lemma 4.6 gives

$$
R_{k}(p, x) \equiv(x-3 p)^{\frac{\ell^{n}-1}{2}} \quad(\bmod \ell) .
$$

Parts (a) and (b) then both follow from Theorem 3.2 when $n$ is odd, we observe that $\frac{\ell^{n}-1}{\ell-1}$ is odd, and so (a) follows from Euler's criterion; when $n$ is even, $\frac{\ell^{n}-1}{\ell-1}$ is even, and so part (b) follows from Fermat's little theorem.

\section{REFERENCES}

[1] S. Ahlgren and K. Ono, A Gaussian hypergeometric series evaluation and Apéry number congruences, J. Reine Angew. Math. 518 (2000), 187-212. MR.1739404 (2001c:11057)

[2] S. Ahlgren, K. Ono, and D. Penniston, Zeta functions of an infinite family of K3 surfaces, Amer. J. Math. 124 (2002), 353-368. MR1890996 (2003e:11068)

[3] S. Frechette, K. Ono, and M. Papanikolas, Gaussian hypergeometric functions and traces of Hecke operators, Int. Math. Res. Not. (2004), no. 60, 3233-3262. MR2096220

[4] F. Q. Gouvêa, Non-ordinary primes: a story, Experiment. Math. 6 (1997), no. 3, 195-205. MR1481589 (99a:11052)

[5] J. Greene, Hypergeometric functions over finite fields, Trans. Amer. Math. Soc. 301 (1987), 77-101. MR0879564 (88e:11122)

[6] D. H. Lehmer, Ramanujan's function $\tau(n)$, Duke Math. J. 10 (1943), 483-492. MR0008619 $(5: 35 \mathrm{~b})$

[7] K. Ono, Values of Gaussian hypergeometric series, Trans. Amer. Math. Soc. 350 (1998), 1205-1223. MR 1407498 (98e:11141)

[8] S. Ramanujan, On certain arithmetical functions, Trans. Camb. Phil. Soc. 22 (1916), 159184.

[9] G. Shimura, Introduction to the arithmetic theory of automorphic functions, Princeton University Press, Princeton, NJ, 1994, Reprint of the 1971 original. MR.1291394 (95e:11048)

[10] H. P. F. Swinnerton-Dyer, Congruence properties of $\tau(n)$, Ramanujan revisited (Urbana, IL, 1987), Academic Press, Boston, MA, 1988, pp. 289-311. MR0938970 (89e:11028)

Department of Mathematics, Texas A\&M University, College Station, Texas 77843

E-mail address: map@math.tamu.edu 